\title{
Case report: Control of intestinal nematodes in captive Chlorocebus sabaeus
}

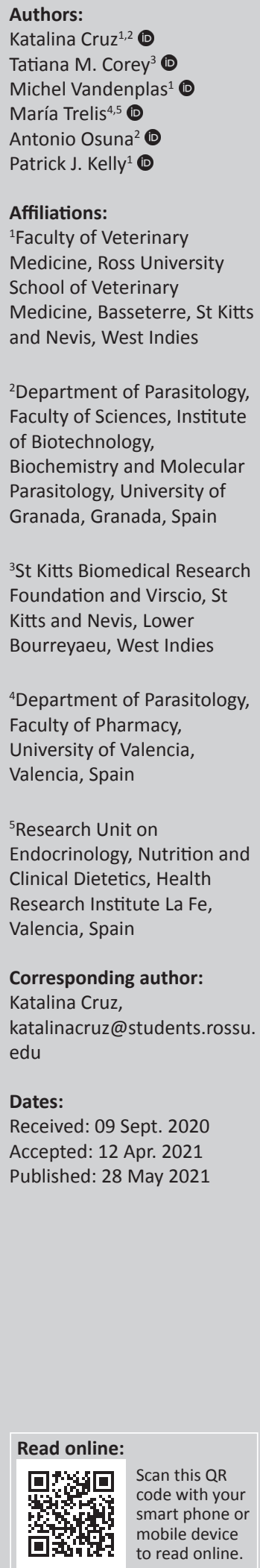

There are limited data on the efficacy of antiparasitic treatments and husbandry methods to control nematode infections in captive populations of African green monkeys (AGMs), Chlorocebus sabaeus. In faecal egg count (FEC) tests, 10 of the 11 (91\%) adult male AGMs captured from the large feral population on the island of St Kitts had evidence of nematode infections, mostly Capillaria (8/11, 73\%), Trichuris trichiura $(7 / 11,64 \%)$ and strongylid species $(7 / 11,64 \%$ ) specifically (hookworm and Trichostrongylus, 50/50), but also Strongyloides fuelleborni $(1 / 11,9 \%)$. When kept in individual cages with cleaning and feeding regimens to prevent reinfections and treated concurrently with ivermectin (300 $\mu \mathrm{g} / \mathrm{kg}$, given subcutaneously) and albendazole (10 $\mathrm{mg} / \mathrm{kg}$, given orally) daily for 3 days, $60 \%(6 / 10)$ of the AGMs were negative at a follow-up FEC at 3 months and by FEC and necropsy at the end of the study 5-8 months later. One monkey appeared to have been reinfected with $T$. trichiura after being negative by FEC at 3 months post-treatment. Four AGMs were positive for T. trichiura at the 3 month FEC follow-up but were negative at the end of the study after one further treatment regimen. Although initially being cleared of Capillaria following treatment, three AGMs were found to be infected at the end of the study. The ivermectin and albendazole treatment regimen coupled with good husbandry practices to prevent reinfections effectively controlled nematode infections in captive AGMs.

Keywords: Capillaria; Trichuris; hookworm; Trichostrongylus; Strongyloides; albendazole; ivermectin; captive; African green monkeys.

\section{Introduction}

Chlorocebus sabaeus, African green monkeys (AGMs) of the Cercopithecidae family, widely found in Africa, were introduced onto the Caribbean island of St Kitts in the 17th century (McGuire 1974). There are limited published data on the parasitic burden of the vast (perhaps 50000; Dore, Gallagher \& Mill 2021 - unpublished) feral population of AGMs, which commonly come into contact with people and may play a role in the epidemiology of human zoonotic parasitic infections common on the island (Berger 2020). Incomplete Caribbean studies have reported island AGMs to be infected with Trichuris trichiura (Yao et al. 2018), Strongyloides sp. (Gallagher et al. 2019; Ritchie et al. 1967), Primasubulura sp. (Cameron 1930) and Schistosoma mansoni (Cameron 1928).

There are limited data on effective anthelmintic treatments for AGMs and husbandry recommendations to control parasitic infections in captive research animals. Nonhuman primate research facilities require effective parasite treatment and prevention protocols to minimise infections that may confound research outcomes and cause unnecessary stress, which lowers the quality of life of study animals. Ritchie et al. (1967) reported that thiabendazole [two $50 \mathrm{mg} / \mathrm{kg}$ doses, PO 14 days apart] significantly reduced or eliminated Strongyloides eggs in the faeces. Kagira et al. (2011) reported that a 3-day regimen of albendazole $(7 \mathrm{mg} / \mathrm{kg}, \mathrm{PO})$ and ivermectin $(300 \mu \mathrm{m} / \mathrm{kg}, \mathrm{SQ})$ reduced the faecal egg counts (FEC) of T. trichiura and strongyles consistently by $100 \%$ after 7,14 and 28 days. Chapman et al. (2016) reported that two doses of ivermectin $(300 \mu \mathrm{g} / \mathrm{kg}, \mathrm{PO}) 5$ days apart resulted in a $100 \%$ cure rate for nematodes in wild AGMs, with no eggs found in the faeces 1 month posttreatment.

How to cite this article: Cruz, K., Corey, T.M., Vandenplas, M., Trelis, M., Osuna, A. \& Kelly, P.J., 2021, 'Case report: Control of intestinal nematodes in captive Chlorocebus sabaeus', Onderstepoort Journal of Veterinary Research 88(1), a1903. https://doi.org/10.4102/ojvr. v88i1.1903

Copyright: @ 2021 . The Authors. Licensee: AOSIS. This work is licensed under the Creative Commons Attribution License. 
Unfortunately, the evaluation of nematode infections is challenging because of inadequate data on the drug efficacy in AGMs and the lack of studies investigating prolonged follow-up, which is needed to detect confounding factors (autoinfection, reinfection, larval transplacental or colostrum transmission, dormant stages and autoinfection resulting from parthenogenesis) (Reichard et al. 2017). Furthermore, FECs can be unreliable, and studies that enable necropsy evaluations are relevant in the absence of a 'gold standard' (Geary et al. 2010).

In this report, we provide a more complete description of the nematode parasites present in AGMs on St Kitts and evaluate a treatment protocol and husbandry practices to control infections in captive AGMs.

\section{Case report}

\section{Animals and facilities}

The St Kitts Biomedical Research Foundation (SKBRF) and translational research affiliate Virscio, Inc. are American Association of Accredited Laboratory Animal Care (AAALAC)-accredited biomedical research facilities for integrated preclinical research and development with a multidisciplinary non-human primate focus. The facility houses around 1000 C. sabaeus study animals according to regulations in the Guide for the Care and Use of Laboratory Animals (National Research Council of the National Academies 2011).

The convenience sample of apparently healthy AGMs used in our study were enrolled in other terminal studies that had been approved by the Institutional Animal Care and Use Committee (approval number AC18175) and conducted in 2019. These studies did not involve procedures that would have influenced the parasitological status of the animals. Eleven wild-caught adult male AGMs were housed in individual stainless steel cages that meet non-human primate requirements (National Research Council of the National Academies 2011) for the duration of their study period. The cages were washed down with water twice daily, and every 2 weeks they were sanitised using a combination of steam $\left(82^{\circ} \mathrm{C}\right)$ and a quaternary-based disinfectant (Consume Eco-Lyzer, Consume Nature's Way, Spartan Chemical Company Inc., Ohio, Maumee, United States [US]), or an accelerated hydrogen peroxide foam (Peroxigard, Virox Technologies Inc., Oakville, Ontario, Canada). Adenosine triphosphate bioluminescence testing of randomly selected dry surfaces was used to determine the sanitation effectiveness.

The main food provided was monkey chow (Envigo Teklad 8773 primate biscuits, Indiana, Indianapolis, US) once a day supplemented with seasonal produce sourced locally. The produce was provided for both nutrition and as an enrichment activity for the animals' well-being, and it was washed first if evidently soiled. Water, provided ad libitum with Lixit water valves, was filtered (Neo-Pure PS-27097-05 9 3/4 inch, 5 microns, NeoLogic Solutions, Greenville, South Carolina, US) and treated with ultraviolet light (Sanitron ultraviolet water purifiers, Atlantic Ultraviolet Corporation, Hauppauge,
New York, US). The water lines were sanitised monthly with a sodium hypochlorite flush delivered by an Edstrom automated watering system (Avidity Science, Waterford, Wisconsin, US).

\section{Treatments}

The deworming regimen was based on a report from Kenya (Kagira et al. 2011) and consisted of ivermectin $(300 \mu \mathrm{g} / \mathrm{kg}$, SQ; Noromectin, Norbrook, Newry, Co. Down, Northern Ireland) and albendazole (IVAL) (10 mg/ $\mathrm{kg}, \mathrm{PO}$ by nasogastric or orogastric gavage; Valbazen, Zoetis, Kalamazoo, Michigan, US) daily for 3 days, following chemical restraint (ketamine $\mathrm{HCl}, 8 \mathrm{mg} / \mathrm{kg}$, Bioniche Pharma USA LLC, US, and xylazine, $1.6 \mathrm{mg} / \mathrm{kg}$, AnaSed, Akorn Inc., Lake Forest, Illinois, US). The animals were returned to their cages and monitored by cage-side observation twice a day for the duration of the study period. No side effects were observed post-treatment. The treatment was repeated if an animal had a positive FEC result during the routine 3 -month screening.

\section{Monitoring of treatments}

Day 0 and 3 month follow-up FECs were carried out on faeces collected directly from the cages. A final FEC and counts of adult $T$. trichiura in the large intestine were performed at routine necropsies 6 or 8 months after the initial IVAL treatment. For the recuperation of adult nematodes, the large intestine was carefully examined whilst removing the ingesta with a circular rubbing motion, washed with $0.9 \%$ saline, filtered through a $100-\mu \mathrm{m}$ sieve and examined for nematodes under a stereomicroscope (7x - 10x magnification).

\section{Faecal analysis and morphologic identification}

For FECs, aliquots ( $2 \mathrm{~g}$ ) of uncontaminated faeces were collected from the cage's under-tray and analysed within $48 \mathrm{~h}$ using the double centrifugation technique (5 min, $500 \mathrm{~g}$ ) with Sheather's sugar solution (spg 1.28). Eggs were identified to the species level whenever possible using described morphological characteristics (Modrý et al. 2015) (Table 1 and Figure 1).

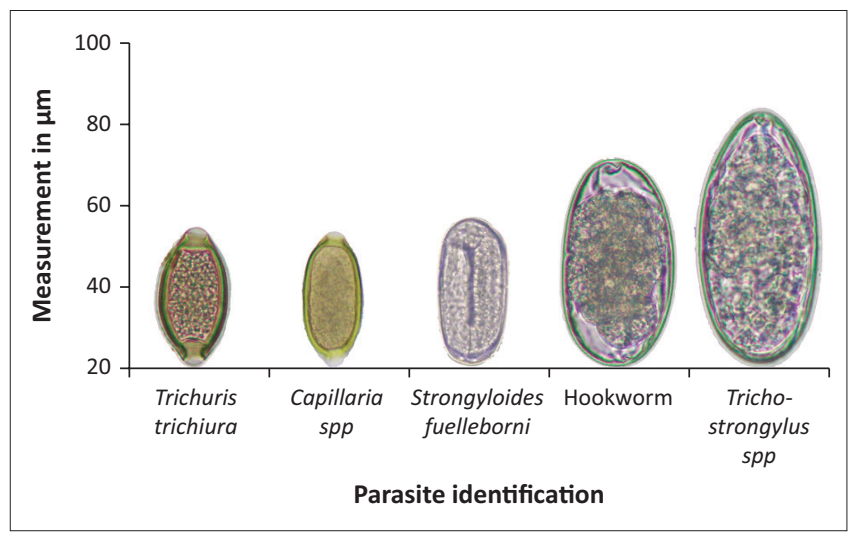

FIGURE 1: Morphologic identification of nematode egg species found (measurements in micrometres). 


\section{Ethical considerations}

The African green monkeys used in our study were enrolled in other terminal studies that had been approved by the Institutional Animal Care and Use Committee of the St Kitts Biomedical Research Foundation. Approval to conduct the study was also obtained from St. Kitts Biomedical Research Foundation, Virscio and Axion Research Foundation, Biomedical Research of the Central Nervous System (IACUC AC18175).

\section{Results and discussion}

Ten of the eleven (91\%) wild-caught adult C. sabaeus study subjects, all male, employed in the study were infected with a variety of nematodes: Capillaria (8/11, 73\%), T. trichiura (7/11, $64 \%$ ), strongylids (7/11, 64\%; hookworms and Trichostrongylus [50/50]) and Strongyloides fuelleborni (S. fuelleborni) (1/11, 9\%) (Tables 2 and 3). Whilst the latter species have been described in AGMs in Africa previously (Chapman et al. 2016; Gallagher et al. 2019; Yao et al. 2018), our finding of Capillaria on the island is of note as eggs of this genus have not previously been reported in the faeces of AGMs.

Infections, then, might have only been acquired in the 300 years since AGMs were introduced onto St Kitts. Unfortunately, we could not determine the Capillaria species involved as egg morphology alone cannot be used for speciation, and local experience is that no adults have been reported in the necropsy findings of AGMs on the island of St. Kitts.

Overall, the IVAL treatment regimen together with the husbandry measures in place in our study were relatively successful at controlling nematode infections in captive AGMs. Following a single IVAL treatment, 6/10 (60\%) of the infected AGMs were negative for nematodes 6-8 months later as confirmed by FEC and at necropsy (Table 2). A second IVAL treatment given to the four AGMs with persistent T. trichiura infections 3 months after the first treatment, resulted in the elimination of the T. trichiura 3-5 months later as evidenced by negative FECs and necropsy findings (Table 3). Although

TABLE 1: Morphologic identification guidelines followed for egg identification

\begin{tabular}{|c|c|c|c|c|c|}
\hline Species & Size $(\mu \mathrm{m})$ & Colour & Shape & Shell & Developmental stage \\
\hline Trichuris trichiura eggs & $50-60 \times 20-30$ & Brown-bile green & Lemon-shaped with bipolar plugs & Smooth & Unembryonated. \\
\hline Capillaria eggs & $50-60 \times 20-30$ & Light yellow/green & $\begin{array}{l}\text { Ovoid barrel-shaped with bipolar } \\
\text { prominences }\end{array}$ & Characteristic striated shell & Unembryonated. \\
\hline Strongyloides fuelleborni eggs & $50-60 \times 30-40$ & Colourless appearance & Ellipsoidal & Thin shell & U-shaped larvae (L1) inside the egg shell. \\
\hline Strongylid eggs & $60-85 \times 35-50$ & Colourless appearance & Ellipsoidal & Smooth and thin shelled & $\begin{array}{l}\text { Hookworms: morula and smaller in size. } \\
\text { Trichostrongylus spp.: early cleavage- } \\
\text { stage embryo, one or both eggshell ends } \\
\text { more pointed, slightly longer. }\end{array}$ \\
\hline
\end{tabular}

Source: Modrý, D., Petrželková, K.J., Kalousová, B. \& Hasegawa, H., 2015, Parasites of African Great Apes, Atlas of coproscopic diagnostics, HPI-Lab, Brno.

TABLE 2: Faecal egg count results and adult Trichuris trichiura found at necropsy in animals treated with ivermectin and albendazole.

\begin{tabular}{|c|c|c|c|c|c|c|c|c|c|c|c|c|c|}
\hline \multirow{2}{*}{$\begin{array}{l}\text { Animal } \\
\text { number }\end{array}$} & \multicolumn{4}{|c|}{ Pretreatment FEC } & \multicolumn{4}{|c|}{ FEC 3 months after one IVAL treatment } & \multicolumn{4}{|c|}{ FEC 6-8 months after one IVAL treatment } & \multirow{2}{*}{$\begin{array}{c}\text { T. trichiura } \\
\text { ibserved in } \\
\text { the large } \\
\text { intestine at } \\
\text { necropsy 6-8 } \\
\text { months after } \\
\text { one IVAL }\end{array}$} \\
\hline & Capillaria & T. trichiura & Strongylids & $\begin{array}{c}S . \\
\text { fuelleborni }\end{array}$ & Capillaria & T. trichiura & Strongylids & $\begin{array}{c}S . \\
\text { fuelleborni }\end{array}$ & Capillaria & T. trichiura & Strongylids & $\begin{array}{c}S . \\
\text { fuelleborni }\end{array}$ & \\
\hline 1 & - & + & + & - & - & - & - & - & - & - & - & - & - \\
\hline 4 & - & + & + & - & - & - & - & - & - & - & - & - & - \\
\hline 6 & + & + & + & - & - & - & - & - & - & - & - & - & - \\
\hline 7 & + & - & + & - & - & - & - & - & - & + & - & - & + \\
\hline 8 & - & - & - & - & - & - & - & - & - & - & - & - & - \\
\hline 10 & + & + & + & - & - & - & - & - & - & - & - & - & - \\
\hline 11 & + & - & - & - & - & - & - & - & - & - & - & - & - \\
\hline
\end{tabular}

Note: Animals that tested negative by faecal egg count 3 months after treatment with ivermectin and albendazole were not re-treated whilst those that were positive were redosed with ivermectin and albendazole.

FEC, faecal egg count; IVAL, ivermectin and albendazole; -, negative; + positive; T. trichiura, Trichuris trichiura; S. fuelleborni, Strongyloides fuelleborni.

TABLE 3: Faecal egg count results and adult Trichuris trichiura found at necropsy in animals treated with ivermectin and albendazole.

\begin{tabular}{|c|c|c|c|c|c|c|c|c|c|c|c|c|c|}
\hline \multirow{2}{*}{$\begin{array}{l}\text { Animal } \\
\text { number }\end{array}$} & \multicolumn{4}{|c|}{ Pretreatment FEC } & \multicolumn{4}{|c|}{ FEC 3 months after first IVAL treatment } & \multicolumn{4}{|c|}{ FEC 3 months after second IVAL treatment } & \multirow{2}{*}{$\begin{array}{l}\text { T. trichiura } \\
\text { observed in the } \\
\text { large intestine } \\
\text { at necropsy } \\
3 \text { months after } \\
\text { second IVAL }\end{array}$} \\
\hline & Capillaria & T. trichiura & Strongylids & $\begin{array}{c}\text { S. } \\
\text { fuelleborni }\end{array}$ & Capillaria & T. trichiura & Strongylids & $\begin{array}{c}S . \\
\text { fuelleborni }\end{array}$ & Capillaria & T. trichiura & Strongylids & $\begin{array}{c}S . \\
\text { fuelleborni }\end{array}$ & \\
\hline 2 & + & + & + & + & - & + & - & - & + & - & - & - & - \\
\hline 3 & + & - & - & - & - & + & - & - & - & - & - & - & - \\
\hline 5 & + & + & - & - & - & + & - & - & + & - & - & - & - \\
\hline Total & $8 / 11(73 \%)$ & $7 / 11(64 \%)$ & ) 7/11 (64\%) & 1/11 (9\%) & - & - & - & - & - & - & - & - & - \\
\hline
\end{tabular}

Note: Animals that tested negative by faecal egg count 3 months after treatment with ivermectin and albendazole were not re-treated, whilst those that were positive were redosed with ivermectin and albendazole.

FEC, faecal egg count; IVAL, ivermectin and albendazole; -, negative; +, positive; T. trichiura, Trichuris trichiura; S. fuelleborni, Strongyloides fuelleborni. 
apparently cleared of Capillaria following the first IVAL treatment, three of these four AGMs had Capillaria eggs in their faeces at necropsy 3-5 months after a second treatment.

The apparent treatment failures with T. trichiura and Capillaria described above warrant further comment. We suspect that the four AGMs that had T. trichiura eggs 3 months after the first treatment with IVAL most likely had infections in the prepatent period, which might exceed 12 weeks in humans (Bundy \& Cooper 1989). It is also possible that the animals became infected whilst in captivity, which is the most likely explanation for the single AGM (Animal 7; Table 2) that was negative for T. trichiura 3 months after the first IVAL treatment but was positive for adults and eggs at necropsy after 8 months. Trichuris eggs can remain inactive on surfaces for years (Bundy \& Cooper 1989; Reichard et al. 2017), and although the AGM cages in our study were cleaned and disinfected regularly, Trichuris eggs are difficult to inactivate (Bundy $\&$ Cooper 1989). It is of note that six of these seven animals that were negative for FEC 3 months after the one IVAL treatment were still negative 6-8 months post-treatment. This indicates that the hygiene measures in use in our facility were mostly effective at preventing reinfection.

How four AGMs became positive for Capillaria following the apparent resolution of infection after the first IVAL treatment is unclear; without being able to determine the infecting specie/s, it is not possible to determine whether these AGMs had been in the prepatent period during treatment, were autoinfected, reinfected or had false-negative test results previously.

The main problem with the IVAL treatment regimen is the requirement for three consecutive sedations for albendazole administration; such repeated dosing, however, is needed for effective ovicidal and larvicidal activity (Martin, Robertson \& Bjorn 1997). Further, this also enabled three administrations of ivermectin, which helps overcome the problem that single doses perhaps only temporarily inhibit the motion and feeding of nematodes instead of causing their death or expulsion (Martin et al. 1997).

\section{Conclusion}

African green monkeys can harbour several nematode species, and effective anthelmintic protocols are needed to ensure the optimal health and well-being of animals in captivity and to prevent zoonotic infections in handlers and local people. The IVAL treatment protocol and husbandry practices we describe would appear to be effective in controlling nematode infections in captive AGMs.

\section{Acknowledgements}

The authors thank the St Kitts Biomedical Research Foundation and Virscio for providing the samples that were analysed for this case report and Monica Gozalbo Monfort and Shamara Welcome for their technical assistance.

\section{Competing interests}

The authors declare that they have no financial or personal relationships that may have inappropriately influenced them in writing this article.

\section{Authors' contributions}

K.C. planned the report, assisted with sample collection, performed the data entry and analysis, and took the lead in writing the manuscript. T.M.C. planned the report, supervised the treatments and sample collection, and critically reviewed the manuscript. M.V., A.O. and M.T. contributed to the student supervision and critically reviewed the manuscript. P.J.K. conceived and planned the report, supervised the execution of the study, and contributed to the interpretation of the results and writing of the manuscript.

\section{Funding information}

Funding was provided by the Ross University School of Veterinary Medicine via the One Health Center for Zoonoses and Tropical Veterinary Medicine.

\section{Data availability}

The authors confirm that the data supporting the findings of this study are available within the article. The raw data were generated at the St Kitts Biomedical Research Foundation and Virscio. Derived data supporting the findings of this study are available from the corresponding author (K.C.) on request.

\section{Disclaimer}

The views expressed in this report are the authors' own and not an official position of the institution or funder.

\section{References}

Berger, D.S., 2020, Infectious diseases of Saint Kitts and Nevis, GIDEON Informatics Inc., Los Angeles, CA.

Bundy, D.A.P. \& Cooper, E.S., 1989, 'Trichuris and Trichuriasis in humans', Advances in Parasitology 28, 107-173. https://doi.org/10.1016/S0065-308X(08)60332-2

Cameron, T.W.M., 1928, 'A new definitive host for Schistosoma mansoni', Journal of Helminthology 6(4), 219-222. https://doi.org/10.1017/\$0022149 X00002364

Cameron, T.W.M., 1930, 'The species of Subulura Molin in primates', Journal of Helminthology 8(1), 49. https://doi.org/10.1017/S0022149X00002510

Chapman, C.A., Friant, S., Godfrey, K., Liu, C., Sakar, D., Schoof, V.A.M. et al., 2016 'Social behaviours and networks of vervet monkeys are influenced by gastrointestinal parasites', PLoS One 11(8), e0161113. https://doi.org/10.1371/ journal.pone.0161113

Dore, K.M., Gallagher, C. \& Mill, C.A., 2021, 'Estimation of green monkey population size in St. Kitts using telemetry and home range analysis', Unpublished manuscript.

Gallagher, C., Beierschmitt, A., Cruz, K., Choo, J. \& Ketzis, J., 2019, 'Should monkeys wash their hands and feet: A pilot-study on sources of zoonotic parasite exposure', One Health 7, 100088. https://doi.org/10.1016/j. parasite exposure',
onehlt.2019.100088

Geary, T.G. Woo, K. McCarthy, J.S., Mackenzie, C.D., Horton, J, Prichard, R.K. et al., 2010, 'Unresolved issues in anthelmintic pharmacology for helminthiases of humans', International Journal for Parasitology 40(1), 1-13. https://doi.org/ 10.1016/j.ijpara.2009.11.001

Kagira, J.M., Mulei, I., Oluoch, G., Waititu, K., Maingi, N. \& Ngotho, M., 2011, 'High efficacy of combined albendazole and ivermectin treatment against gastrointestinal nematodes in vervet monkeys and baboons', Scandinavian gastrointestinal nematodes in vervet monkeys and
Journal of Laboratory Animal Science 38(3), 187-193. 
Martin, R.J., Robertson, A.P. \& Bjorn, H., 1997, 'Target sites of anthelmintics', Parasitology 114(Suppl 7), S111-S124. https://doi.org/10.1017/S0031182097001029

McGuire, M., 1974, 'The St. Kitts vervet', Contributions to primatology, vol. 1 , Karger, Basel.

Modrý, D., Petrželková, K.J., Kalousová, B. \& Hasegawa, H., 2015, Parasites of African Great Apes, Atlas of coproscopic diagnostics, HPI-Lab, Brno.

Reichard, M.V., Thomas, J.E., Chavez-Suarez, M., Cullin, C.O., White, G.L., Wydysh, E.C. et al., 2017, 'Pilot study to assess the efficacy of ivermectin and fenbendazole for treating captive-born olive baboons (Papio anubis) coinfected with Strongyloides fülleborni and Trichuris trichiura, Journal of the American Association for Laboratory Animal Science 56(1), 52-56.
Ritchie, L.S., Knight, W.B., Oliver-Gonzalez, J., Frick, L.P., Morris, J.M. \& Croker, W.L., 1967, 'Schistosoma mansoni infections in Cercopithecus sabaeus monkeys', The Journal of Parasitology 53(6), 1217-1224. https://doi.org/10. 2307/3276683

National Research Council of the National Academies, 2011, Guide for the care and use of laboratory animals, 8th edn., National Academies Press, Washington, DC.

Yao, C., Walkush, J., Shim, D., Cruz, K. \& Ketzis, J., 2018, 'Molecular species identification of Trichuris trichiura in African green monkey on St. Kitts, West Indies', Veterinary Parasitology: Regional Studies and Reports 11, 22-26. https://doi.org/10.1016/j.vprsr.2017.11.004 